\title{
ON THE DOMINATOR CHROMATIC NUMBER OF THE GENERALIZED CATERPILLARS FOREST
}

\author{
Soumia Aioula ${ }^{1}$, Mustapha Chellali $^{2}$ And Noureddine Ikhlef-Eschouf ${ }^{3, *}$
}

\begin{abstract}
A dominator coloring is a proper coloring of the vertices of a graph such that each vertex of the graph dominates all vertices of at least one color class (possibly its own class). The dominator chromatic number of a graph $G$ is the minimum number of color classes in a dominator coloring of $G$. In this paper, we determine the exact value of the dominator chromatic number of a subclass of forests which we call, generalized caterpillars forest, where every vertex of degree at least three is a support vertex.
\end{abstract}

Mathematics Subject Classification. 05C15, 05C69.

Received March 20, 2020. Accepted October 24, 2020.

\section{INTRODUCTION}

Throughout the paper, we consider finite, simple and undirected graphs. Let $G$ be a graph with vertex-set $V(G)$ and edge-set $E(G)$. The open neighborhood of a vertex $v \in V(G)$ is the set $N(v)=\{u \mid u v \in E\}$. The degree of a vertex $v$ is $d_{G}(v)=|N(v)|$. A vertex of degree one is called a leaf, and its neighbor is called a support vertex. Given a subset $A \subseteq V(G)$, we denote by $G[A]$ (or sometimes $G \backslash A$ ) the subgraph of $G$ induced by $A$.

Recall that a tree is a connected acyclic graph, and a forest is an acyclic graph. A caterpillar is a tree such that the removal of all its leaves produces a path.

A coloring of the vertices of $G$ is a mapping $c: V(G) \rightarrow \mathbb{N}$, where for every vertex $v$ the integer $c(v)$ is called the color of $v$. A coloring $c$ is proper if for any two adjacent vertices $u$ and $v, c(v) \neq c(u)$. The chromatic number $\chi(G)$ of graph $G$ is the smallest integer $k$ such that $G$ admits a proper coloring with $k$ colors. Let $X$ be a color class of a proper coloring of $G$. Then we say that a vertex $x$ of $G$ sees $X$ if $x$ is adjacent to all vertices in $X$, and $x$ misses $X$ otherwise. In particular, if $X=\{x\}$, then we say that $x$ sees its own class.

A dominator coloring of $G$ is a proper coloring of the vertices of $G$ such that each vertex in $G$ sees at least one color class (possibly its own class). The dominator chromatic number $\chi_{d}(G)$ is the minimum number of color classes in a dominator coloring of $G$. A dominator coloring of $G$ with $\chi_{d}(G)$ colors will be called a $\chi_{d}$-coloring of $G$. The concept of dominator coloring was introduced by Gera et al. [6] and studied further by Gera $[4,5]$, Chellali and Maffray [3] and Boumediene and Chellali [1,2]. In particular, in [2] the authors gave a polynomial

\footnotetext{
Keywords. Dominator coloring, dominator chromatic number, trees.

1 Laboratory of Mechanics, Physics and Mathematical Modeling, Faculty of Sciences, University of Médéa, Médéa, Algeria.

2 LAMDA-RO Laboratory, Department of Mathematics, University of Blida, B.P. 270, Blida, Algeria.

3 Department of Mathematics and Computer Science, Faculty of Sciences, University of Médéa, Médéa, Algeria.

*Corresponding author: nour_echouf@yahoo.fr
} 
time algorithm computing the dominator chromatic number for every nontrivial tree. It is worth noting that the decision problem corresponding to the dominator coloring is NP-complete for arbitrary graphs [6]. Therefore, it is natural to look for graph classes where the value of the dominator chromatic number is given either exactly or can be computed in polynomial time.

Given a $\chi_{d}$-coloring $c$ of $G$, we denote by $\Omega_{c}$ the set of color classes of $c$ containing a single vertex, and let $\Pi_{c}$ be the set of the remaining color classes of $c$. Let us also define the following sets.

- Let $\Pi_{c}^{1}$ be the subset of color classes that are missed, that is $\Pi_{c}^{1}=\left\{X \in \Pi_{c} \mid\right.$ no vertex of $G$ sees $\left.X\right\}$, and let $\Pi_{c}^{2}=\Pi_{c} \backslash \Pi_{c}^{1}$.

- For $i \in\{1,2\}$, let $B_{c}^{i}$ be the set of all vertices belonging to color classes in $\Pi_{c}^{i}$, and let $A_{c}=V(G) \backslash\left(B_{c}^{1} \cup B_{c}^{2}\right)$.

Clearly $A_{c}, B_{c}^{1}, B_{c}^{2}$ are disjoint sets and $V(G)=A_{c} \cup B_{c}^{1} \cup B_{c}^{2}$. Also, $\left|A_{c}\right|=\left|\Omega_{c}\right|$ and $\left|B_{c}^{1} \cup B_{c}^{2}\right| \geq 2\left|\Pi_{c}\right|$.

It has been shown in [1] that for every $\chi_{d}$-coloring $c$ of a nontrivial tree either each support vertex belongs to $A_{c}$ or its unique leaf neighbor belongs to $A_{c}$. Moreover, they proved the following.

Proposition $1.1([1])$. Every tree of order at least three admits a $\chi_{d^{-}}$coloring $c$ such that each support vertex belongs to $A_{c}$ and all leaves of $G$ have the same color.

In this paper, we are interested in determining the exact value of the dominator chromatic number for a more general class of caterpillars which we call generalized caterpillars. A generalized caterpillar is a tree such that each vertex of degree at least three is a support vertex. A generalized caterpillars forest is a forest such that each component is a generalized caterpillar. A stalk in a generalized caterpillar forest $G$ is a path whose endvertices are support vertices in $G$ and whose inner vertices are not. Clearly, each stalk (if any) has order at least two. Also, if $G$ is a generalized caterpillar forest without stalks, then each component of $G$ is a star or a single vertex.

It is worth mentioning that every tree $T$ of order at least three is a subtree of a generalized caterpillar. Indeed, it is enough to add for any vertex of degree at least 3 that is not a support vertex a new vertex attached to it. Clearly, in this way the supertree obtained, which will denoted by $G_{T}$, is a generalized caterpillar. In this context, if $T$ is a tree of order $n \geq 3$, then $I_{T}$ will denote the set of vertices of degree at least three that are not support vertices. Obviously, if $T$ is a tree with $I_{T}=\emptyset$, then $G_{T}=T$. Our next observation gives a relationship between $\chi_{d}(T)$ and $\chi_{d}\left(G_{T}\right)$ for every nontrivial tree $T$.

Observation 1.2. If $T$ is a nontrivial tree, then $\chi_{d}\left(G_{T}\right)-\left|I_{T}\right| \leq \chi_{d}(T) \leq \chi_{d}\left(G_{T}\right)$.

Proof. Clearly, if $T$ has order two or $I_{T}$ is empty, then $G_{T}=T$ and the result is valid. Hence we can assume that $T$ has order at least three and $I_{T} \neq \emptyset$. The upper bound follows from the fact that the restriction of any $\chi_{d}$-coloring of $G_{T}$ to $T$ is a dominator coloring of $T$. Now to prove the lower bound, consider a $\chi_{d}$-coloring $c$ of $T$ satisfying Proposition 1.1. Let $M=I_{T} \backslash A_{c}$ and $\pi$ be a coloring of $G_{T}$ obtained from $c$ as follows. Color each vertex of $M$ with a new, different color; and color the new vertices in $G_{T}$ with the color used by the leaves in $T$. The remaining vertices of $G_{T}$ keep their colors already given by coloring $c$. It is easy to see that $\pi$ is a dominator coloring of $G_{T}$ with $\chi_{d}(T)+|M|$ colors, and thus $\chi_{d}\left(G_{T}\right) \leq \chi_{d}(T)+|M| \leq \chi_{d}(T)+\left|I_{T}\right|$.

The sharpness of the bounds in Observation 1.2 is given by the following result.

Observation 1.3. For every integer $j \geq 0$, there exists a tree $T_{j}$ such that $\left|I_{T_{j}}\right|=j$ and $\chi_{d}\left(G_{T_{j}}\right)=\chi_{d}\left(T_{j}\right)+\left|I_{T_{j}}\right|$.

Proof. Clearly, if $j=0$, then for any caterpillar $T$ we have $G_{T}=T$ and thus $\chi_{d}\left(G_{T}\right)=\chi_{d}(T)$. Hence let $j \geq 1$ be an integer. Let $H_{i}$ be a tree obtained from a star $K_{1,3}$ centered at $u_{i}$ by subdividing each edge exactly once, and let $v_{i}$ be a support vertex of $H_{i}$. Let $T_{j}$ be a tree obtained from $H_{1}, H_{2}, \ldots, H_{j}$ by adding $j-1$ edges connecting $v_{i}$ 's so that they induce a path $P_{j}$. For example, the tree $T_{3}$ is illustrated in Figure 1 . A tree $T_{3}$ and its corresponding generalized caterpillar $G_{T 3}$. Note that $T_{j}$ has $3 j$ support vertices, and since the remaining vertices of $T_{j}$ that are independent, we deduce from Proposition 1.1 that $\chi_{d}\left(T_{j}\right)=3 j+1$. Moreover, the generalized caterpillar $G_{T_{j}}$ constructed from $T_{j}$ by adding for each $u_{i}$ a new vertex attached to it by an edge contains $4 j$ support vertices. One can easily see that $\chi_{d}\left(G_{T_{j}}\right)=4 j+1=\chi_{d}\left(T_{j}\right)+j$. 


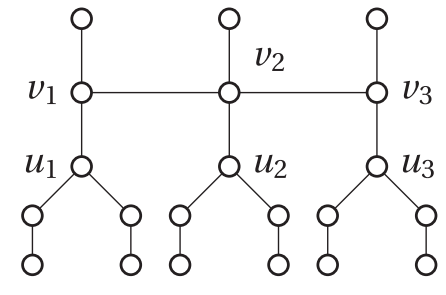

$\mathrm{T}_{3}$

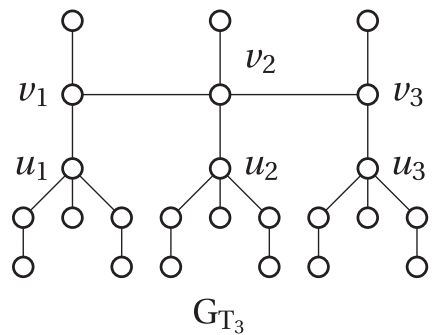

FIGURE 1. A tree $\mathrm{T}_{3}$ and its corresponding generalized caterpillar $\mathrm{G}_{\mathrm{T}_{3}}$

By using a similar proof to that presented in [1], we can see that a generalized caterpillar forest $G$ admits a $\chi_{d}$-coloring $c$ such that each support vertex belongs to $A_{c}$ and all leaves of $G$ have the same color. Hence we have the following.

Corollary 1.4. Proposition 1.1 is still valid for generalized caterpillar forest.

Observation 1.5. Let $G$ be a generalized caterpillar forest with $p \geq 0$ single vertices and $q \geq 0$ nontrivial stars, and let $H$ be the subgraph of $G$ containing all components that are neither single vertices nor stars. Then $\chi_{d}(G)=p+q+\chi_{d}(H)+i$, where $i=1$ if $q \geq 1$ and $V(H)$ is empty, and $i=0$ otherwise.

Proof. If $G$ contains no edge, then clearly $\chi_{d}(G)=p$. Hence assume that $G$ contains at least one edge. If $V(H)$ is empty, then it is easy to show that $\chi_{d}(G)=p+q+1$.

From now on, we can assume that $V(H)$ is non-empty. Let $G_{1}, G_{2}, \ldots, G_{r}$ be the components of $G-H$ (if any). Clearly $\chi_{d}(H) \geq 2$, and $\chi_{d}\left(G_{i}\right) \leq 2$ since $G_{i}$ is a single vertex or a nontrivial star. This means that $\chi_{d}(H) \geq \chi_{d}\left(G_{i}\right)$ for every $i$. Moreover, each component in $G-H$ needs at least one new color, since a vertex in such component must see an entire color class. Hence $\chi_{d}(G) \geq p+q+\chi_{d}(H)$.

The equality follows by exhibiting a dominator coloring of $G$ with $p+q+\chi_{d}(H)$ colors. According to Corollary 1.4, $H$ admits a $\chi_{d^{-}}$-coloring $c$ such that all leaves have the same color, say 1 . Let $\pi$ be a coloring of $G$ defined as follows. For every $x \in V(H)$, let $\pi(x)=c(x)$, and for each leaf $v$ in $G-H$, let $\pi(v)=1$, unless $v$ belongs to a component of order 2, in which case $v$ is one of the two leaves. Color the remaining vertices of $G$ differently using $(p+q)$ new colors. Clearly, $\pi$ is a dominator coloring using $p+q+\chi_{d}(H)$ colors, and thus $\chi_{d}(G) \leq p+q+\chi_{d}(H)$.

According to Observation 1.5, we can assume in the remainder of this paper that each component of a generalized caterpillar forest is nontrivial and different from a star. Our aim is to prove the following result.

Theorem 1.6. Let $G$ be a generalized caterpillar forest with $s$ support vertices and $p$ connected components, each is nontrivial and different from a star. Let $n_{i} \geq 2$ be the order of the $i^{\text {th }}$ stalk of $G$. Then

$$
\chi_{d}(G)=\alpha+s+\sum_{i=1}^{s-p}\left\lfloor\frac{n_{i}-2}{3}\right\rfloor,
$$

where $\alpha= \begin{cases}1 & \text { if each } n_{i} \in\{2,3,5\} \\ 2 & \text { otherwise }\end{cases}$ 


\section{Proof of Theorem 1.6}

The proof of Theorem 1.6 is based on the following preliminary results.

Observation 2.1. Let $G$ be a generalized caterpillar forest such that each component is nontrivial and different from a star. Then $G$ admits a $\chi_{d}$-coloring $c$ such that the following properties hold.

(i) All leaves of $G$ are in $B_{c}^{1}$ and hence every vertex in $A_{c} \cup B_{c}^{2}$ has degree at least two.

(ii) Every vertex in $B_{c}^{1}$ has degree at most two.

(iii) Every vertex in $B_{c}^{2}$ has degree exactly two.

Proof. Let $c$ be a $\chi_{d}$-coloring satisfying Corollary 1.4.

(i) Let $X$ be the color class containing all leaves of $G$. Since $G$ has at least one stalk, each vertex in $G$ misses $X$, and the first part of item (i) follows. The second part of item (i) is obvious.

(ii) If $B_{c}^{1}$ has a vertex of degree at least three, then such a vertex would be a support vertex, contradicting the choice of $c$.

(iii) Using the fact that every vertex of degree at least three is a support vertex, the desired result follows from item (i).

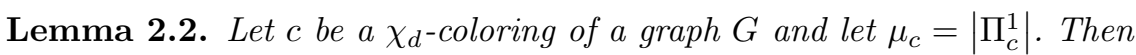

(i) $\mu_{c} \leq \chi(G)$. In particular, if $G$ is bipartite, then $\mu_{c} \in\{0,1,2\}$.

(ii) Moreover, if $G$ is a generalized caterpillar forest such that each component is nontrivial and different from a star, then $\mu_{c} \in\{1,2\}$.

Proof. (i) Suppose to the contrary that $\mu_{c} \geq \chi(G)+1$, that is, at least $\chi(G)+1$ colors appear in $B_{c}^{1}$. Without loss of generality, we may assume that vertices in $B_{c}^{1}$ use colors $1,2, \ldots, \mu_{c}$. Since no color can appear in both $B_{c}^{1}$ and $V(G) \backslash B_{c}^{1}$, vertices in $V(G) \backslash B_{c}^{1}$ must use the remaining colors, that is, colors $\mu_{c}+1, \ldots, \chi_{d}(G)$. Define a new coloring $\pi$ of $G$ as follows. Recolor properly all vertices of $B_{c}^{1}$ with $\chi\left(G\left[B_{c}^{1}\right]\right)$ colors among $\left\{1,2, \ldots, \mu_{c}\right\}$ (this is possible since $\chi\left(G\left[B_{c}^{1}\right]\right) \leq \chi(G) \leq \mu_{c}-1$ ), while vertices of $V(G) \backslash B_{c}^{1}$ keep their colors already given by $c$. Clearly $\pi$ is a dominator coloring of $G$ with $\chi_{d}(G)-\mu_{c}+\chi\left(G\left[B_{c}^{1}\right]\right)<\chi_{d}(G)$ colors, a contradiction. The second part of item (i) follows from the fact that $\chi(G) \leq 2$ for bipartite graphs.

(ii) Note first that $\mu_{c} \neq 0$, since $B_{c}^{1}$ is nonempty (by Observation 2.1(i)). This together with Lemma 2.2(i) yield the desired result.

Lemma 2.3. Let $G$ be a generalized caterpillar forest such that each component is nontrivial and different from

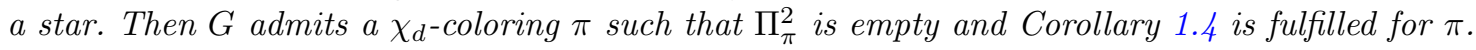

Proof. Among all $\chi_{d}$-colorings of $G$ fulfilling Corollary 1.4, let $c$ be chosen so that

(C1) $\left|\Omega_{c}\right|$ is maximized.

(C2) Subject to (C1), $\left|\Pi_{c}^{1}\right|$ is maximized.

Put $k=\chi_{d}(G)$ and $\mu_{c}=\left|\Pi_{c}^{1}\right|$. Let $X_{1}, X_{2}, \ldots, X_{k}$ be the color classes of $c$ in which vertices in $X_{i}$ get color $i$ for $i \in\{1, \ldots, k\}$. Assume to the contrary that $\Pi_{c}^{2}$ is nonempty. Pick $t$ from $\{1, \ldots, k\}$ such that $X_{t} \in \Pi_{c}^{2}$ and let $X_{t}=\left\{x_{1}, x_{2}, \ldots, x_{p}\right\}$. By definition, $p \geq 2$ and each $x_{i} \in B_{c}^{2}$. Also, by Observation 2.1(iii), we have

$$
d_{G}\left(x_{i}\right)=2 \text { for every } i \in\{1, \ldots, p\} .
$$

Let us denote by $W\left(X_{t}\right)$ the set of vertices of $G$ that see $X_{t}$. Then, since $G$ is acyclic, we have

$$
\left|W\left(X_{t}\right)\right|=1 \text {. }
$$


Thus, let $W\left(X_{t}\right)=\left\{w_{t}\right\}$. Clearly,

$$
d_{G}\left(w_{t}\right) \geq p \geq 2
$$

In view of $(2.1)$, let $N_{G}\left(x_{i}\right)=\left\{w_{t}, y_{i}\right\}$ for $i \in\{1, \ldots, p\}$ and put $Y_{t}=\left\{y_{1}, y_{2}, \ldots, y_{p}\right\}$. Obviously $N_{G}\left(X_{t}\right)=Y_{t} \cup\left\{w_{t}\right\}$. Observe that, since $G$ is acyclic, $N_{G}\left[X_{t}\right]$ induces a subdivided star of order $2 p+1$ centered at $w_{t}$.

Recall that Lemma 2.2(ii) shows that $\mu_{c} \in\{1,2\}$. Without loss of generality, we can assume that $\Pi_{c}^{1}=\left\{X_{1}\right\}$ when $\mu_{c}=1$ and $\Pi_{c}^{1}=\left\{X_{1}, X_{2}\right\}$ when $\mu_{c}=2$. In this case, we have $t \geq \mu_{c}+1$ (since $X_{t} \in \Pi_{c}^{2}$ ). Let $\pi$ be a $\chi_{d}$-coloring of $G$ obtained from $c$ and defined according to the following cases.

Case 1. $w_{t} \in B_{c}^{1} \cup B_{c}^{2}$.

Items (ii) and (iii) of Observation 2.1 together with (2.3) yield $d_{G}\left(w_{t}\right)=2$. Then $p=2$ and $N_{G}\left(X_{t}\right)=\left\{w_{t}, y_{1}, y_{2}\right\}$. Thus $N_{G}\left(X_{t}\right)$ induces a path $P_{5}: y_{1}-x_{1}-w_{t}-x_{2}-y_{2}$. Next, we will show that $y_{1}$ and $y_{2}$ either are both in $A_{c}$ or one of them is in $A_{c}$ and the other one is in $B_{c}^{2}$ having a neighbor in $A_{c}$. In each case, we will recolor some vertices of $G$ and increase the number of color classes of $\Omega_{c}$ or $\Pi_{c}^{1}$. To this end, consider the following situations whether $w_{t}$ is in $B_{c}^{1}$ or $B_{c}^{2}$.

Subcase 1. $w_{t} \in B_{c}^{1}$. Note that since $d_{G}\left(x_{1}\right)=d_{G}\left(x_{2}\right)=2$, neither $x_{1}$ nor $x_{2}$ can see a color class of $\Pi_{c}^{2}$, for otherwise, one of them will be of degree at least 3, which contradicts (2.1). Therefore, both $y_{1}$ and $y_{2}$ must be in $A_{c}$, for otherwise, one of $x_{1}$ and $x_{2}$ misses all color classes of $c$, which contradicts the definition of $c$. Define $\pi$ as follows: assign color 1 to $x_{1}$ and $x_{2}$, and assign color $t$ to $w_{t}$. The remaining vertices of $G$ keep their colors (given by $c$ ). It is easy to see that $\pi$ is a $\chi_{d}$-coloring of $G$ fulfilling Corollary 1.4 such $\Omega_{\pi}=\Omega_{c} \cup\left\{w_{t}\right\}$ (that is, $\Omega_{\pi}$ contains more color classes than $\Omega_{c}$ ), which contradicts the choice of $c$.

Subcase 2. $w_{t} \in B_{c}^{2}$. Let $X_{s}=\left\{x_{1}^{\prime}, x_{2}^{\prime}, \ldots, x_{q}^{\prime}\right\}$ be the color class containing $w_{t}=x_{1}^{\prime}$. Clearly $t \neq s \geq \mu_{c}+1$. Since $X_{s} \in \Pi_{c}^{2},(2.2)$ says that $W\left(X_{s}\right)=\left\{w_{s}\right\}$. If $w_{s} \notin\left\{x_{1}, x_{2}\right\}$, then $d_{G}\left(w_{t}\right) \geq 3$, contradicting the fact that $d_{G}\left(w_{t}\right)=2$. Hence, $w_{s} \in\left\{x_{1}, x_{2}\right\}$, say $w_{s}=x_{1}$. Since $w_{s}$ and $X_{s}$ play the same role as $w_{t}$ and $X_{t}$, respectively, we conclude that $q=2$, and each vertex in $X_{t} \cup X_{s}$ has degree 2. Thus $x_{2}^{\prime}=y_{1}$ and $X_{s}=\left\{w_{t}, y_{1}\right\}$. Also, since $d_{G}\left(y_{1}\right)=2$, there is a vertex $z_{1} \neq x_{1}$ such that $y_{1} z_{1} \in E(G)$. Hence $N_{G}\left(X_{t} \cup X_{s}\right)$ induces a path $P_{6}: z_{1}-y_{1}-x_{1}-w_{t}-x_{2}-y_{2}$. Using the same argument as in Subcase 1 , we can see that $y_{2}, z_{1} \in A_{c}$. Now assigning color 1 to $x_{2}$ and $y_{1}$ and keep the colors already given to the remaining vertices (under $c$ ) provides a $\chi_{d}$-coloring $\pi$ of $G$ fulfilling Corollary 1.4 such that $\Omega_{\pi}=\Omega_{c} \cup\left\{\left\{x_{1}\right\},\left\{w_{t}\right\}\right\}$, which contradicts again the choice of $c$.

Case 2. $w_{t} \in A_{c}$.

Then $c\left(w_{t}\right) \neq 1$. We claim that $Y_{0}=Y_{t} \cap B_{c}^{1}$ is nonempty. Suppose to the contrary that $Y_{0}=\emptyset$. Then each vertex in $Y_{t}$ has color different from 1 . By recoloring all vertices of $X_{t}$ with color 1 (this is possible since $t \geq \mu_{c}+1$ ), we would obtain a dominator coloring of $G$ with $\chi_{d}(G)-1$, a contradiction, which proved the claim. Now, let $y_{i_{0}}$ be any vertex in $Y_{0}$. By Observation 2.1(ii), $y_{i_{0}}$ has degree at most two. If $d_{G}\left(y_{i_{0}}\right)=1$, then $y_{i_{0}}$ misses all color classes of $c$, which is impossible. So $d_{G}\left(y_{i_{0}}\right)=2$ and let $N_{G}\left(y_{i_{0}}\right)=\left\{x_{i_{0}}, z_{i_{0}}\right\}$. A similar argument as to the previous cases shows that vertex $z_{i_{0}}$ is in $A_{c}$. In this case, we define $\pi$ by interchanging colors of $x_{i_{0}}$ and $y_{i_{0}}$ and keeping the same colors for the remaining vertices of $G$. Clearly, $\pi$ is a $\chi_{d^{-}}$-coloring of $G$ fulfilling Corollary 1.4. In addition, $\Omega_{\pi}=\Omega_{c}$, but $\Pi_{\pi}^{1}=\Pi_{c}^{1} \cup\left\{\left(X_{t} \backslash\left\{x_{i_{0}}\right\}\right) \cup\left\{y_{i_{0}}\right\}\right.$, which contradicts the choice of $c$.

In the rest of this paper, we denote by $T_{i}$ the $i^{\text {th }}$ stalk of order $n_{i} \geq 2$ in the generalized caterpillar forest $G$, where $V\left(T_{i}\right)=\left\{x_{1}^{i}, x_{2}^{i}, \ldots, x_{n_{i}-1}^{i}, x_{n_{i}}^{i}\right\}$ and $x_{j}^{i} x_{j+1}^{i} \in E(G)$ for every $j \in\left\{1,2, \ldots, n_{i}-1\right\}$. We also denote by $I_{i}=\left\{x_{2}^{i}, x_{3}^{i}, \ldots, x_{n_{i}-1}^{i}\right\}$ (possibly empty) the set of inner vertices in $T_{i}$.

Lemma 2.4. Let $G$ be a generalized caterpillar forest such that each component is nontrivial and different from a star. If $c$ is a $\chi_{d}$-coloring fulfilling the statement of Lemma 2.3, with $\mu_{c}=\left|\Pi_{c}^{1}\right|$, then

(i) If $\left|I_{i}\right| \geq 3$, then for every three consecutive vertices of $I_{i}$, one of them belongs to $B_{c}^{1}$ and another to $A_{c}$.

(ii) If $\mu_{c}=1$ and $\left|I_{i}\right| \geq 2$, then one of any two consecutive vertices of $I_{i}$ belongs to $A_{c}$.

(iii) $x_{1}^{i}, x_{n_{i}}^{i} \in A_{c}$. 
Proof. We first observe that by Lemma 2.3, each vertex of $G$ is in $B_{c}^{1} \cup A_{c}$.

(i) Let $\left|I_{i}\right| \geq 3$, and suppose to the contrary, that for some $j_{0} \in\left\{2, \ldots, n_{i}-3\right\}$ all of $x_{j_{0}}^{i}, x_{j_{0}+1}^{i}, x_{j_{0}+2}^{i}$ belong to $A_{c}$. Then, recoloring $x_{j_{0}+1}^{i}$ with a color used by the leaves provides a dominator coloring of $G$ with $\chi_{d}(G)-1$ colors, a contradiction. Therefore, for every $j \in\left\{2, \ldots, n_{i}-3\right\}$, one of $x_{j}^{i}, x_{j+1}^{i}, x_{j+2}^{i}$ belongs to $B_{c}^{1}$. Moreover, one of $x_{j_{0}}^{i}, x_{j_{0}+1}^{i}, x_{j_{0}+2}^{i}$ must be in $A_{c}$, for otherwise, these three vertices will be all in $B_{c}^{1}$ and thus $x_{j_{0}+1}^{i}$ misses all color classes of $c$, which is impossible.

(ii) Follows from the fact that each vertex of $G$ is in $B_{c}^{1} \cup A_{c}$.

(iii) Follows from the definition of the stalk $T_{i}$ and Corollary 1.4.

Lemma 2.5. Let $G$ be a generalized caterpillar forest with $r \geq 1$ stalks $T_{1}, \ldots, T_{r}$, s support vertices and $p$ components each is nontrivial and different from a star. Consider a $\chi_{d}$-coloring $c$ of $G$ satisfying Lemma 2.3 and $\mu_{c}$ be the number of colors of $c$ appearing in $G\left[B_{c}^{1}\right]$. Then

(i) $\chi_{d}(G)=s+\mu_{c}+\sum_{i=1}^{r}\left\lfloor\frac{\left|V\left(T_{i}\right)\right|-2}{3}\right\rfloor$ with $r=s-p$.

(ii) If $\left|V\left(T_{i}\right)\right| \in\{2,3,5\}$ for each $i \in\{1, \ldots, r\}$, then $\mu_{c}=1$. Otherwise, $G$ admits a $\chi_{d^{-c o l o r i n g}} \varphi$ such that $\mu_{\varphi}=2$.

Proof. According to Lemma 2.3, $\Pi_{c}^{2}$ is empty and Corollary 1.4 is fulfilled for $c$. Hence $\chi_{d}(G)=\left|\Pi_{c}^{1}\right|+\left|\Omega_{c}\right|$. Also, since $\left|\Pi_{c}^{1}\right|=\mu_{c}$ and $\left|\Omega_{c}\right|=\left|A_{c}\right|$, we get

$$
\chi_{d}(G)=\mu_{c}+\left|A_{c}\right| .
$$

Let $n_{i}=\left|V\left(T_{i}\right)\right|$ and recall that $V\left(T_{i}\right)=\left\{x_{1}^{i}, x_{2}^{i}, \ldots, x_{n_{i}}^{i}\right\}$ and $I_{i}=V\left(T_{i}\right) \backslash\left\{x_{1}^{i}, x_{n_{i}}^{i}\right\}$. Let $S_{G}$ be the set of support vertices of $G$ and $\left|S_{G}\right|=s$. Clearly,

$$
A_{c}=S_{G} \cup\left(\cup_{i=1}^{r} I_{i} \cap A_{c}\right)
$$

and

$$
n_{i}=2+\left|I_{i} \cap B_{c}^{1}\right|+\left|I_{i} \cap A_{c}\right| .
$$

(i) It is easy to cheek that $r=s-p$. Hence, combining this together with (2.4) and (2.5), we obtain

$$
\chi_{d}(G)=\mu_{c}+s+\sum_{i=1}^{s-p}\left|I_{i} \cap A_{c}\right| .
$$

In the sequel, we shall show that

$$
\left|I_{i} \cap A_{c}\right|=\left\lfloor\frac{n_{i}-2}{3}\right\rfloor \text { for all } i .
$$

To do this, we need to prove the following three claims. Let $\lambda_{c}^{i}$ denote the number of colors of $c$ appearing in $G\left[I_{i} \cap B_{c}^{1}\right]$.

Claim 2.6. If $n_{i}=2$, then $\lambda_{c}^{i}=0$, while if $n_{i} \geq 3$, then $1 \leq \lambda_{c}^{i} \leq \mu_{c} \leq 2$.

Proof. Clearly, by (2.6), $n_{i} \geq 2$. If $n_{i}=2$, then $I_{i}=\emptyset$ and thus $\lambda_{c}^{i}=0$. Hence, assume that $n_{i} \geq 3$. Then $I_{i} \neq \emptyset$. If $\lambda_{c}^{i}=0$, then all vertices of $I_{i}$ are in $A_{c}$. In this case, recoloring one of these vertices with a color used by the leaves provides a dominator coloring of $G$ with $\chi_{d}(G)-1$ colors, a contradiction. Therefore $\lambda_{c}^{i} \geq 1$. Now, using the fact that $\mu_{c} \in\{1,2\}$ (by Lem. 2.2(ii)), and the definition of $\lambda_{c}^{i}$ we obtain $\lambda_{c}^{i} \leq \mu_{c} \leq 2$. This achieves the proof of Claim 2.6.

In what follows, we can assume, without loss of generality, that vertices in $B_{c}^{1}$ use either colors 1 and 2 when $\mu_{c}=2$ or only color 1 when $\mu_{c}=1$. We will additionally assume that all leaves are colored with color 1 . 
Claim 2.7. If $\mu_{c}=2$, then for all $i \in\{1,2, \ldots, r\}$ either $n_{i} \in\{2,3,5\}$ or $\lambda_{c}^{i}=2$.

Proof. Suppose, to the contrary, that there is an integer $i_{0} \in\{1,2, \ldots, r\}$ such that $n_{i_{0}} \notin\{2,3,5\}$ and $\lambda_{c}^{i_{0}} \neq 2$. By Claim 2.6, $\lambda_{c}^{i_{0}}=1$. Let $I_{i_{0}}=\left\{x_{2}^{i_{0}}, x_{3}^{i_{0}}, \ldots, x_{n_{i_{0}}-1}^{i_{0}}\right\}$, and observe that every vertex of $I_{i_{0}}$ not colored with 1 uses a color belonging to $A_{c}$ (because of $\lambda_{c}^{i_{0}}=1$ ), and thus

$$
\left|I_{i_{0}} \cap A_{c}\right|=\left\lfloor\frac{n_{i_{0}}-2}{2}\right\rfloor .
$$

Since $\mu_{c}=2$, we can define a dominator coloring $\varphi$ of $G$ obtained from $c$ as follows. For each $x \notin I_{i_{0}}$, $\varphi(x)=c(x)$; for each $i \equiv 2(\bmod 3)$, let $\varphi\left(x_{i}^{i_{0}}\right)=1$; for each $i \equiv 0(\bmod 3)$, let $\varphi\left(x_{i}^{i_{0}}\right)=2$; for the remaining vertices of $I_{i_{0}}$, we color them differently among colors used by $I_{i_{0}} \cap A_{c}$. Clearly now under $\varphi$, each vertex of $I_{i_{0}}$ not colored with 1 or 2 uses a color belonging to $A_{\varphi}$, and thus

$$
\left|I_{i_{0}} \cap A_{\varphi}\right|=\left\lfloor\frac{n_{i_{0}}-2}{3}\right\rfloor .
$$

Now, since $\left|I_{i_{0}} \cap A_{\varphi}\right|<\left|I_{i_{0}} \cap A_{c}\right|, \varphi$ is a dominator coloring of $G$ using less colors than $c$, which leads to a contradiction. This achieves the proof of Claim 2.7.

Claim 2.8. If $n_{i_{0}} \notin\{2,3,5\}$ for some $i_{0} \in\{1,2, \ldots, r\}$, then $G$ admits a $\chi_{d^{-}}$coloring with $\mu=2$.

Proof. If $\mu_{c}=2$, we are done. Hence assume that $\mu_{c}=1$ and thus, by Claim 2.6, $\lambda_{c}^{i_{0}}=1$. First, assume that $n_{i}=4$ for each $i$. Define a new dominator coloring $\pi$ as follows: color each support vertex with a new color starting from 3, and color all its neighbors by colors 1 or 2 so that both colors appear in each stalk. Clearly, $|\pi|=|c|$ and $\mu_{\pi}=2$. For the next, we can assume that $n_{i} \geq 6$ for at least some $i$. Without loss of generality, we can assume that color 2 appears in $I_{i_{0}} \cap A_{c}$. Let $\varphi$ be the dominator coloring of $G$ defined as in Claim 2.7 with $|\varphi|$ colors. A similar argument as in Claim 2.6 shows that (2.9) and (2.10) remain valid. In addition, we have

$$
\mu_{\varphi}=\mu_{c}+1 \text { and }\left|I_{i} \cap A_{\varphi}\right|=\left|I_{i} \cap A_{c}\right| \text { for all } i \neq i_{0}
$$

and

$$
|\varphi|=\mu_{\varphi}+s+\left|I_{i_{0}} \cap A_{\varphi}\right|+\sum_{i=1\left(i \neq i_{0}\right)}^{s-p}\left|I_{i} \cap A_{\varphi}\right| .
$$

By replacing the expressions of (2.11) together with (2.10) and (2.9) in (2.12), we obtain

$$
|\varphi|=\mu_{c}+s+1+\left\lfloor\frac{n_{i_{0}}-2}{3}\right\rfloor-\left\lfloor\frac{n_{i_{0}}-2}{2}\right\rfloor+\sum_{i=1}^{s-p}\left|I_{i} \cap A_{c}\right| .
$$

Using (2.7), we get

$$
|\varphi|=1+\left\lfloor\frac{n_{i_{0}}-2}{3}\right\rfloor-\left\lfloor\frac{n_{i_{0}}-2}{2}\right\rfloor+\chi_{d}(G) .
$$

Now, since $\left\lfloor\frac{n_{i_{0}}-2}{3}\right\rfloor \leq\left\lfloor\frac{n_{i_{0}}-2}{2}\right\rfloor-1$, it follows that $|\varphi| \leq \chi_{d}(G)$. That is $\varphi$ is $\chi_{d}$-coloring of $G$ with $\mu_{\varphi}=2$. This achieves the proof of Claim 2.8.

Now we turn our attention to prove equality (2.8). Pick $i_{0}$ from $\{1,2, \ldots, r\}$ and consider the following cases.

Case 1. $n_{i_{0}} \in\{2,3,5\}$.

If $n_{i_{0}}=2$, then $I_{i_{0}}=\emptyset$ and thus (2.8) holds since $\left|I_{i_{0}} \cap A_{c}\right|=0$. If $n_{i_{0}}=3$, then clearly $\left|I_{i_{0}}\right|=1$. In this case, again $\left|I_{i_{0}} \cap A_{c}\right|=0$, for otherwise recoloring the vertex of $I_{i_{0}}$ with a color used by the leaves provides 
a dominator coloring of $G$ with $\chi_{d}(G)-1$ colors, a contradiction. Thus (2.8) holds for $n_{i_{0}}=3$. Finally, let $n_{i_{0}}=5$. Then $\left|I_{i_{0}}\right|=3$, where two vertices of $I_{i_{0}}$ do not belong to $A_{c}$ (for otherwise recoloring the two non-adjacent vertices of $I_{i_{0}}$ with the color used by the leaves and the other vertex with a color already used by $I_{i_{0}} \cap A_{c}$ provides a dominator coloring of $G$ with $\chi_{d}(G)-1$ colors, a contradiction). Thus $\left|I_{i_{0}} \cap A_{c}\right| \leq 1$. This together with Lemma 2.4(i) yield $\left|I_{i_{0}} \cap A_{c}\right|=1$ and thus (2.8) holds for $n_{i_{0}}=5$.

Case 2. $n_{i_{0}} \notin\{2,3,5\}$.

By Claim 2.8, we may assume that $\mu_{c}=2$ and thus by Claim 2.7, we have $\lambda_{c}^{i_{0}}=2$. Moreover, we have $\left|I_{i_{0}} \cap B_{c}^{1}\right| \geq 2$. This together with (2.6) imply that $n_{i_{0}} \geq 4$. Consider the dominator coloring $\varphi$ of $G$ as defined in the proof of Claim 2.7. Note that (2.10) and (2.12) remain valid. In addition, we have

$$
\mu_{\varphi}=\mu_{c} \text { and }\left|I_{i} \cap A_{\varphi}\right|=\left|I_{i} \cap A_{c}\right| \text { for all } i \neq i_{0} .
$$

Now, by substituting the expressions of (2.10) and (2.13) in formula (2.12), we obtain

$$
|\varphi|=\mu_{c}+s+\left\lfloor\frac{n_{i_{0}}-2}{3}\right\rfloor+\sum_{i=1\left(i \neq i_{0}\right)}^{s-p}\left|I_{i} \cap A_{c}\right| .
$$

Now, since $|\varphi| \geq \chi_{d}(G),(2.14)$ and (2.7) together yield $\left|I_{i_{0}} \cap A_{c}\right| \leq\left\lfloor\frac{n_{i_{0}}-2}{3}\right\rfloor$.

On the other hand, Lemma 2.4(i) yields $\left|I_{i_{0}} \cap A_{c}\right| \geq\left\lfloor\frac{n_{i_{0}}-2}{3}\right\rfloor$, and thus equality (2.8) follows. This achieves the proof of Item (i).

(ii) Assume that $n_{i} \in\{2,3,5\}$ for all $i \in\{1,2, \ldots, r\}$ and let $l$ be the number of stalks of order 5 . In this case, we have $\sum_{i=1}^{s-p}\left\lfloor\frac{n_{i}-2}{3}\right\rfloor=l$. By replacing this in the expression of item (i), we get

$$
\chi_{d}(G)=\mu_{c}+s+l .
$$

Now, let $\psi$ be a coloring of $G$ obtained from $c$ as follows. For each $I_{i}$, let $\psi\left(x_{j}^{i}\right)=1$ if $j$ is even, and color the vertices $x_{j}^{i}$ with $j$ odd differently among colors used by $I_{i} \cap A_{c}$. The remaining vertices of $G$ keep their colors given by coloring $c$. It is easy to show that $\psi$ is dominator coloring using at least $\chi_{d}(G)$ colors such that $\mu_{\psi}=1$ and $\left|I_{i} \cap A_{\psi}\right|=\left\lfloor\frac{n_{i}-2}{2}\right\rfloor$ for each $i$, where

$$
|\psi|=1+s+\sum_{i=1}^{s-p}\left\lfloor\frac{n_{i}-2}{2}\right\rfloor .
$$

Using the fact that $n_{i} \in\{2,3,5\},(2.16)$ becomes

$$
|\psi|=1+s+l
$$

Since $|\psi| \geq \chi_{d}(G)$ and $\mu_{c} \in\{1,2\},(2.15)$ and (2.17) together yield $\mu_{c}=1$.

The second part follows by Claim 2.8. This completes the proof of Lemma 2.5.

Now, we are ready to prove Theorem 1.6.

Proof of Theorem 1.6. If $n_{i} \in\{2,3,5\}$ for all $i$, then by items (i) and (ii) of Lemma 2.5, we have $\mu_{c}=1$ and thus $\chi_{d}(G)=s+1+\sum_{i=1}^{s-p}\left\lfloor\frac{n_{i}-2}{3}\right\rfloor$. Now if $n_{i} \notin\{2,3,5\}$ for some $i$, then by the second part of Lemma 2.5(ii), we can take $\mu_{c}=2$. Using Lemma 2.5(i), we obtain $\chi_{d}(G)=s+2+\sum_{i=1}^{s-p}\left\lfloor\frac{n_{i}-2}{3}\right\rfloor$. 


\section{REFERENCES}

[1] H. Boumediene Merouane and M. Chellali, On the dominator colorings in trees. Discuss Math. Graph Theory 32 (2012) 677-683.

[2] H. Boumediene Merouane and M. Chellali, An algorithm for the dominator chromatic number of a tree. J. Combin. Optim. 30 (2015) 27-33.

[3] M. Chellali and F. Maffray, Dominator colorings in some classes of graphs. Graphs Combin. 28 (2012) $97-107$.

[4] R. Gera, On dominator colorings in graphs. Graph Theory Notes New York 52 (2007) 25-30.

[5] R. Gera, On the dominator colorings in bipartite graphs. In: Proceedings of the 4th International Conference on Information Technology: New Generations (ITNG) (2007) 947-952.

[6] R. Gera, S. Horton and C. Rasmussen, Dominator colorings and safe clique partitions. Congr. Numer. 181 (2006) $19-32$. 